\title{
Pattern Projection with a Sinusoidal Phase Grating
}

\author{
Elena Stoykova, Jana Harizanova, and Ventseslav Sainov \\ Central Laboratory of Optical Storage and Processing of Information, Bulgarian Academy of Sciences, \\ P.O. Box 95, 1113 Sofia, Bulgaria
}

Correspondence should be addressed to Elena Stoykova, elena@optics.bas.bg

Received 1 October 2007; Accepted 21 March 2008

Recommended by John Watson

\begin{abstract}
The aim of this work is to study the diffractive properties of a sinusoidal phase grating for incorporation as a pattern projection element in a multisource and multicamera phase-shifting profilometric system. Two challenges should be overcome for successful operation of such a system, which are connected to inherent limitations of the phase-shifting algorithm-requirements for a sinusoidal fringe profile and for equal background and contrast of fringes in the recorded patterns. As a first task, we analyze the frequency content of the projected fringes in the Fresnel diffraction zone for parallel and divergent light illumination at different grating parameters and wavelengths. As a second task, we evaluate the systematical errors due to higher harmonics and multiwavelength illumination. Finally, operation of the four-wavelength profilometric system is simulated, and the error of the profilometric measurement evaluated. The results of test measurements are also presented.
\end{abstract}

Copyright (c) 2009 Elena Stoykova et al. This is an open access article distributed under the Creative Commons Attribution License, which permits unrestricted use, distribution, and reproduction in any medium, provided the original work is properly cited.

\section{Introduction}

Parallel acquisition of information as an inherent property of optical methods stimulated development of various optical techniques for noncontact measurement of surfaces of 3D objects and scenes. Among existing techniques, the methods which rely on a functional relationship of the sought object data with the phase of a periodic fringe pattern (FP) projected onto and reflected from the object occupy a special place as a metrological means with noncomplex setups and processing algorithms that are suitable for outdoor implementation $[1,2]$.

Phase retrieval from a deformed FP, recorded as a function of spatial coordinates and time, requires solution of a nonlinear inverse problem based on fringe analysis of a fringe profile [2]:

$$
I(\vec{r}, t)=I_{0}(\vec{r}, t)+I_{V}(\vec{r}, t) f[\varphi(\vec{r}, t)+\phi(\vec{r}, t)],
$$

where $I_{0}(\vec{r}, t)$ is a slowly varying background intensity, $I_{V}(\vec{r}, t)$ is a fringe visibility which is also a low-frequency signal, $f[\cdots]$ is a periodic function, $f \in[-1,1]$, and $\varphi(\vec{r}, t)$ is a phase term related to the measured parameter, for example, an object profile. The additional phase term $\phi(\vec{r}, t)$ is optional, being introduced during the formation of the waveform $f$ or during the phase evaluation process.
As the phase retrieval involves nonlinear operations, many algorithms need some constraints to be applied.

Over the years, a host of phase retrieval algorithms has been proposed and tested [3]. From the point of view of $3 \mathrm{D}$ object capture, an ideal algorithm must ensure real-time automatic precise calculation of the phase from generally wideband FPs, which are recorded from complicated objects with large depth variation and discontinuities such as steps, holes, and protrusions. Real-time operation can be done by phase retrieval from a single pattern (single-shot acquisition) or from multiple patterns which are recorded at high acquisition speed and processed by simple fast algorithms [4]. Using Fourier transform [5-7] is a straightforward way for phase retrieval from a single pattern, but it requires introduction of a high carrier frequency within the FP and also suffers from limitation on object height variation. In addition, due to the global character of the transform, the reconstructed surface is influenced by imperfections within the whole FP. The approaches based on space-frequency representations $[8,9]$ for phase retrieval from a single pattern are still under development. Recently, the so-called spatial analysis methods for phase retrieval from a single pattern have been reported as regularized phase tracking which involves time-consuming iterative procedures to solve a set of linear equations $[10,11]$ : fitting-error modified spatial 
fringe modulation $[12,13]$, phase demodulation based on fringe skeletonizing when an extreme map is introduced by locating the fringes minima and maxima $[14,15]$, phasestepping recovery of objects by numerical generation of multiple frames from a single recorded frame [16]. However, spatial methods usually rely on a slow phase variation within the FP, which hampers analysis of wideband FPs, or requires time-consuming iterations. As it seems, fast acquisition of multiple FPs is a more effective technical solution for the realtime capture.

In the case of $M$ recorded FPs, $I_{m}(x, y), m=1,2, \ldots, M$, which are equally shifted at a phase step $\phi$, the object phase is determined from the expression $[2,17]$

$$
\varphi(x, y)=\arctan \frac{\sum_{m=1}^{M} b_{m} I_{m}(x, y)}{\sum_{m=1}^{M} a_{m} I_{m}(x, y)},
$$

where the coefficients $a_{m}, b_{m}$ depend on the phase step $\phi$. This phase-shifting technique is a very popular pointwise approach for phase demodulation due to its high accuracy and large dynamic range. It is widely applied in its temporal modification with successive recording of the FPs in time, thus being inapplicable for a real-time capture.

Recently, there have been some reports on real-time recording/processing of phase-shifted patterns [4, 18-20]. A single-shot measurement by simultaneous projection of three colour patterns (red, green, and blue) on the object at different angles and Fourier analysis of the deformed image recorded by a single CCD camera are described in $[18,19]$. A phase-stepping method for measuring the 3D surface profile of a moving object by projection of a sinusoidal grating pattern and continuous intensity acquisition by three phaseshifted linear array sensors positioned along the projected stripes is proposed in [20]. The method is restricted to objects moving at a constant speed. High-resolution 3D measurement of absolute coordinates using three phaseshifted fringe patterns coded with three primary colors and recorded at data acquisition speed of $90 \mathrm{fps}$ is presented in [4].

In [21], we proposed a technical solution of a single-shot pattern projection profilometric system with simultaneous projection and recording of four phase-shifted FPs which are generated at four different wavelengths. The system includes a pattern projection module with four projection elements irradiated by four near-infrared diode lasers and a registration module with four CCD cameras. As candidates for a projection element, a sinusoidal phase grating and a holographic optical element which reconstructs two point sources have been discussed [21]. Technical simplicity of setup, easy manufacturing, reproducibility of the desired modulation and spacing, high efficiency, minimization of the phase-shifting error, and independence of the spatial period of the diffraction pattern on the wavelength advocated strongly the choice of the phase grating. This solution determines the aim of this work which is to study the diffractive properties of a sinusoidal phase grating for incorporation as a pattern projection element in a multisource and multicamera profilometric system. Two challenges should be overcome for successful operation of such a system, which are connected to inherent limitations of the phaseshifting algorithm-requirements for a sinusoidal fringe profile and for equal background and contrast of fringes in the recorded FPs. As a first task, we analyze the frequency content of the projected fringes in the Fresnel diffraction zone for parallel and divergent light illumination at different grating parameters and wavelengths. As a second task, we evaluate the systematical errors due to higher harmonics and multiwavelength illumination. Finally, operation of the fourwavelength profilometric system is simulated, and the error of the profilometric measurement is evaluated. The results of test measurements are also presented.

\section{Four-Wavelength Pattern Projection System}

Optical arrangement of the four-wavelength profilometric system is presented in Figure 1. The fringe pattern generation module consists of 4 blocks corresponding to four different wavelengths $\left(\lambda_{1}-\lambda_{4}\right)$ as shown in Figure 1, where DL1-DL4 are $20 \mathrm{~mW}$ CW single-mode diode lasers, and G1-G4 are identical sinusoidal phase gratings. The diode lasers emit in NIR spectral region at wavelengths of $\lambda_{1}=790 \mathrm{~nm}, \lambda_{2}$ $=810 \mathrm{~nm}, \lambda_{3}=850 \mathrm{~nm}$, and $\lambda_{4}=910 \mathrm{~nm}$. The pattern generation module ensures simultaneous projection of four sinusoidal patterns of equal spacing that are phase-shifted at $\pi / 2$. To optimize the optical efficiency of wavelength mixing, the different interference mirrors (IM1-IM3) are used as follows: the mirror IM1 transmits $\lambda_{1}$ and reflects $\lambda_{2}$, the mirror IM2 transmits $\lambda_{1}, \lambda_{2}$ and reflects $\lambda_{3}, \lambda_{4}$, and the mirror IM3 transmits $\lambda_{3}$ and reflects $\lambda_{4}$. The object is illuminated by the four patterns simultaneously using a projection objective.

The registration module consists of four synchronized CCD cameras for simultaneous capture of the FPs which have been deformed by the measured surface. The spectral separation of the individual patterns is provided by a second set of interference mirrors, that is, IM1-IM3. The recorded FPs are further processed by a four-step phase-shifting algorithm with $M=4$ and $\phi=\pi / 2$ in (2). The precise positioning and adjustments of cameras and optical elements are required to avoid possible systematic errors and to ensure parallel recording of the phase-shifted patterns, modulated by the object surface.

\section{Frequency Content of Diffraction Pattern from a Sinusoidal Phase Grating}

3.1. Theory. If the phase-shifting algorithm (2) is applied for phase retrieval, violation of the assumption $f[\cdots]=$ $\cos (\cdots)$ in (1) leads to systematic errors in the evaluated phase. Pure sinusoidal fringes can be created by twobeam interference in an interferometer. However, inevitable environmental noise and complexity of the setup jeopardize outdoor application of such a system. This motivates the implementation of other approaches for sinusoidal pattern projection such as using spatial light modulators [22] or 


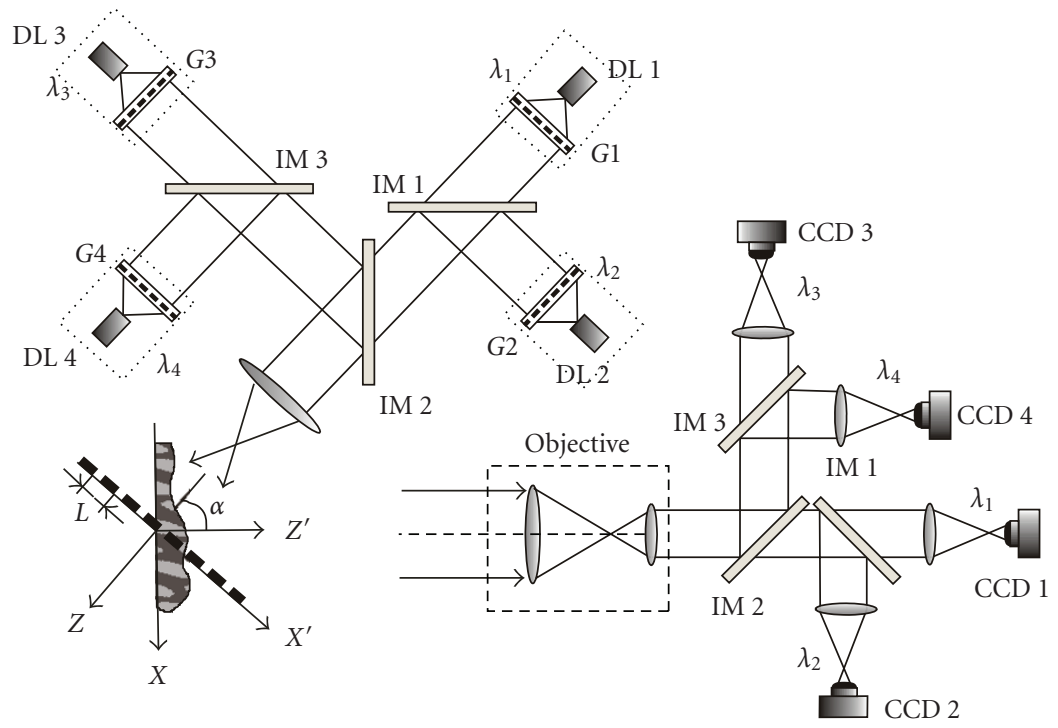

FIGURE 1: Optical arrangement of the four-wavelength pattern projection system-DL1-4: diode lasers, G1-4: diffraction gratings, IM1-3: interference mirrors.

diffraction gratings [23], however at the expense of higher harmonics in the projected pattern.

Over the years, the systematic errors caused by the nonlinearity of phase-shifters and recording devices or by the presence of higher harmonics have been comprehensively studied $[23,24]$ and, as a result, rather elaborated errorcompensating algorithms have been proposed. The shortcoming of these algorithms is their controversial behavior: the more efficient they are in suppressing the systematic error, the more vulnerable they become to random errors. Quite often, the solution is found in presuming that the higher harmonics are negligible in the projected pattern [24], but one could hardly take this assumption for granted without analysis of the frequency content of the projected fringes. For example, higher harmonics are unavoidable in the fringe profile $f(x, y)$ created by a defocused Ronchi grating which is a popular way to generate sinusoidal fringes $[25,26]$ :

$$
f(x, y)=a_{0}+\sum_{q=1}^{Q} \sin c\left(\frac{\pi q}{2}\right) \frac{J_{1}(2 \pi q b / 2)}{\pi q b / 2} \cos \left(\frac{2 \pi q x}{L}\right)
$$

where $a_{0}$ is the average value, $Q$ is the number of harmonics, $L$ is the grating spacing, and $b$ is defocusing parameter; the grating lines are parallel to the $y$-axis.

A thin sinusoidal phase grating located in the $(X, Y)$ plane with grating lines parallel to the $y$-axis is characterized with transmittance [27]:

$$
\tau(x, y)=\exp \left[j m \sin \left(2 \pi \frac{x}{L}\right)\right]=\sum_{q=-\infty}^{\infty} J_{q}(m) \exp \left(j 2 \pi q \frac{x}{L}\right),
$$

where $m$ is the modulation parameter, $L$ is the grating spacing along the $x$-axis, and $J_{q}$ is a Bessel function of the first kind (order $q$ ). At illumination with a unit-amplitude normally incident plane wave, the Fresnel diffraction pattern at distance $z$ behind the grating is a structure that is periodic along the directions $x$ and $z$. A complex amplitude $U(x, y, z)$ in this case can be found using the transfer function approach [26], with the transfer function being valid for Fresnel diffraction:

$$
H\left(f_{x}, f_{y}\right)=\exp (j k z) \exp \left[-j \pi \lambda z\left(f_{x}^{2}+f_{y}^{2}\right)\right]
$$

which yields

$$
\begin{aligned}
& U(x, y, z) \\
& =J_{0}(m)+\sum_{q=1}^{\infty}\left[J_{q}(m) \exp \left(j \frac{2 \pi q x}{L}\right)+J_{-q}(m)\right. \\
& \left.\quad \exp \left(-j \frac{2 \pi q x}{L}\right)\right] \exp \left(-j \frac{\pi \lambda z q^{2}}{L^{2}}\right) \\
& =-J_{0}(m)+2 \sum_{q=0}^{\infty} J_{2 q}(m) \cos \left[\frac{4 q \pi x}{L}\right] \\
& \quad\left\{\cos \left[\frac{(2 q)^{2} \pi \lambda z}{L^{2}}\right]-j \sin \left[\frac{(2 q)^{2} \pi \lambda z}{L^{2}}\right]\right\} \\
& +j J_{2 q+1}(m) \sin \left[\frac{(2 q+1) 2 \pi x}{L}\right] \\
& \left\{\cos \left[\frac{(2 q+1)^{2} \pi \lambda z}{L^{2}}\right]-j \sin \left[\frac{(2 q+1)^{2} \pi \lambda z}{L^{2}}\right]\right\} .
\end{aligned}
$$

In the above expressions, $\lambda$ is the wavelength, and $f_{x}, f_{y}$ are the spatial frequencies in the plane of observation which is parallel to the grating and located at distance $z$ from it. The 
factor $\exp (j k z)$ is omitted as representing a constant phase delay at all spatial frequencies, and the equality $J_{-q}(m)=$ $(-1)^{q} J_{q}(m)$ is taken into account. The finite extent of the grating aperture is neglected. As can be seen, the intensity distribution $I(x, y, z)=U U^{*}$ obtained from the expression (6) is not sinusoidal. The amplitudes of different harmonics and hence the profile of the projected fringes along the $x$ - and $z$-axes depend on the grating spacing $(L)$, the wavelength $(\lambda)$, and the modulation parameter $(m)$.

Along the $x$ - and $z$-axes, the diffraction pattern consists of alternating zones with a phase-reversed contrast. Along the $x$-axis, due to the limited aperture of the objectives used for projection and recording of fringes, we can freely assume that we project a part of the diffraction pattern that contains only fringes with equal contrast. The fact that the fringe spacing does not depend on the wavelength can be used to illuminate the object by spatially similar fringes at different wavelengths in order to record simultaneously the deformed FPs by separate CCD cameras and to overcome the main drawback of the temporal phase-shifting profilometry in which acquisition of patterns is made successively in time.

The spatial period of the first harmonic along the $x$ axis depends only on the grating spacing. The spatial period of the first harmonic along the $z$-axis depends on all three parameters $L, \lambda$, and $m$. The alternating zones with a phasereversed contrast along the $z$-axis are located between the planes which correspond to

$$
\begin{gathered}
z_{n}=\frac{2 n L^{2}}{\lambda}, \quad n=0,1,2, \ldots, \\
z_{n}^{\prime}=\frac{(2 n+1) L^{2}}{\lambda}, \quad n=0,1,2, \ldots
\end{gathered}
$$

The planes located at $z_{n}$ and $z_{n}^{\prime}, n=1,2, \ldots$, contain a "perfect" grating image with uniform intensity distribution equal to unity. These planes correspond to Talbot images [27]. The length of the zone along the $z$-axis with a phaseconstant contrast of fringes for the first harmonic is equal to $d_{z 1}=z_{n}^{\prime}-z_{n}=L^{2} / \lambda$, respectively. The length of this zone for the second harmonic is $d_{z 2}=L^{2} / 2 \lambda$, and for the $q$ th harmonic it is $d_{z q}=L^{2} / q \lambda$. This means that the second harmonic is eliminated in the planes parallel to the grating and located at

$$
z_{n}^{\prime \prime}=\left(2 n+\frac{1}{2}\right) \frac{L^{2}}{\lambda}, \quad n=0,1,2, \ldots
$$

However, the amplitude of the third harmonic is maximal in these planes. As should be expected, the modulation parameter $m$ affects intensely the frequency content of the diffraction pattern. We evaluated its influence in the interval from 0.1 up to 1.6. The first-order harmonic dominates at all values of $m$. The degrading effect of higher-order harmonics becomes unacceptable at $m$, being greater than 0.3. The zones that are close to the distances, $z=z_{n}^{\prime \prime}$, $n=0,1,2, \ldots$, appear to be optimal for fringe projection because, at least theoretically, the energy in the first harmonic reaches the maximum for $m$, up to $0.6-0.8$ at $z=z_{n}^{\prime \prime}$, whereas the second harmonic is missing. As it seems, by a proper choice of the modulation parameter $(m)$ and the distance

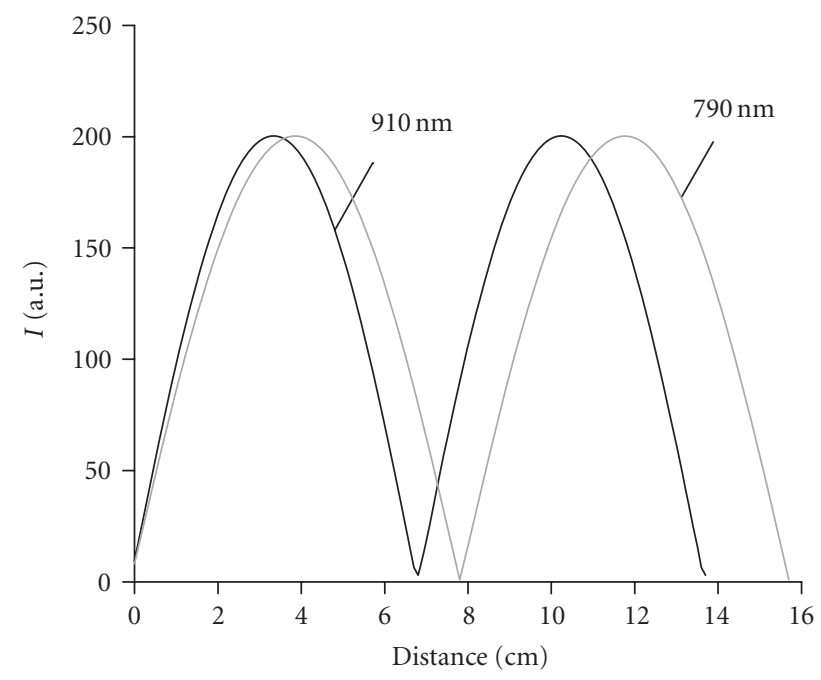

FIGURE 2: Spatial variation of energy concentrated in the first harmonic along the $z$-axis in the Fresnel zone at plane-wave illumination of a sinusoidal phase grating.

$(z)$, the influence of the higher diffraction orders could be minimized. However, location of these optimal planes along the $z$-axis varies with the wavelength. Figure 2 depicts spatial variation of the energy concentrated in the first harmonic along the $z$-axis for the wavelengths $790 \mathrm{~nm}$ and $910 \mathrm{~nm}$ for a grating spacing $L=0.025 \mathrm{~cm}$ and $m=0.2$.

Obviously, the difference between the values of $z_{n}^{\prime \prime}$ corresponding to wavelengths $\lambda_{1}, \lambda_{2}$ increases as

$$
z_{n}^{\prime \prime}\left(\lambda_{1}\right)-z_{n}^{\prime \prime}\left(\lambda_{2}\right)=\left(2 n+\frac{1}{2}\right) L^{2}\left(\frac{1}{\lambda_{1}}-\frac{1}{\lambda_{2}}\right), \quad n=0,1,2, \ldots,
$$

along with the distance from the grating. In practical realization of the system, the four gratings are located at the same distance from the projective lens. This means that it will be difficult to ensure the same contrast and spectral content of the FPs projected at different wavelengths if the distance between the gratings and the projective lens is too large. Theoretically, at $L=0.25 \mathrm{~cm}, z_{0}^{\prime \prime}(790 \mathrm{~nm})-z_{0}^{\prime \prime}(910 \mathrm{~nm})=$ $0.52 \mathrm{~cm}$, whereas $z_{1}^{\prime \prime}(790 \mathrm{~nm})-z_{1}^{\prime \prime}(910 \mathrm{~nm})=2.6 \mathrm{~cm}$. If the distance from the grating is chosen within the above intervals and the modulation parameter is kept low, one could expect a practically sinusoidal profile of the same contrast for fringes projected at 790, 810,850, and $910 \mathrm{~nm}$. This conclusion is confirmed by Figure 3 which gives the intensity distribution and the spatial frequency spectrum as a function of the wavelength at $z=10.5 \mathrm{~cm}$.

A phase diffraction grating at coherent illumination creates fringes which are focused at any distance from the grating. However, rapidly varying contrast of the fringes due to the Talbot effect makes it impossible for pattern projection profilometry to have large-scale objects at planewave illumination. Indeed, the distance between the Talbot planes at plane-wave illumination is on the order of several centimeters. To increase the spatial zone of equal contrast, the grating should be illuminated by a divergent beam. If 


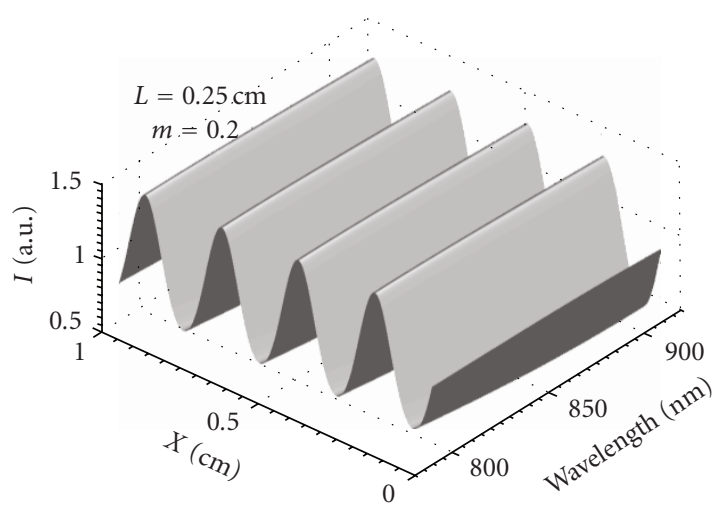

(a)

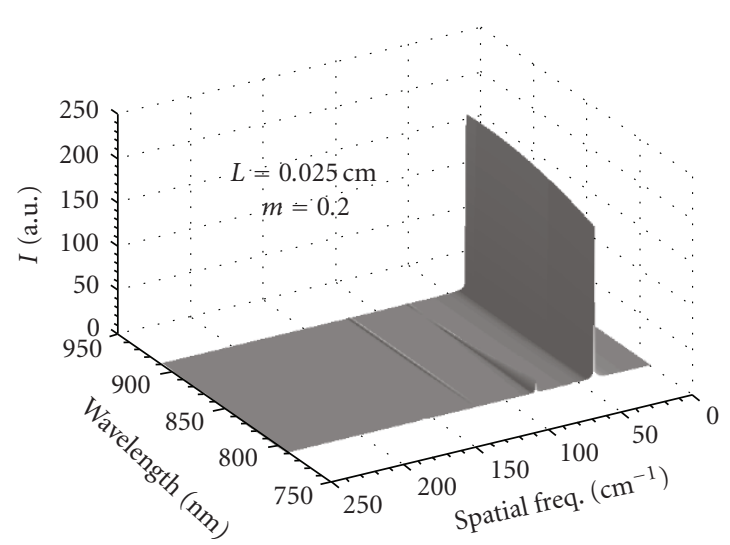

(b)

FIGURE 3: The intensity distribution and spatial frequency spectrum of the light transmitted by a sinusoidal phase grating with $L=$ $0.025 \mathrm{~cm}$ in the Fresnel zone as a function of the wavelength at $z=10.5 \mathrm{~cm}$ for a plane-wave illumination. For convenience, the part of the spectrum at the fundamental frequency is omitted.

this beam is created by a point source at distance $s$ from the grating, the fringe spacing is magnified according to the geometrical formula (similar triangles):

$$
\Lambda(z)=1+\frac{z}{s},
$$

where $\Lambda$ denotes the magnification. In this case, the Talbot planes of grating self-imaging can be found at [28]

$$
\frac{1}{z_{T}}+\frac{1}{s}=\frac{\lambda}{n L^{2}}
$$

where $z_{T}$ is the $z$-coordinate of a Talbot plane, and $n$ is an integer. Figure 4 gives the intensity distribution behind the grating with $L=0.025 \mathrm{~cm}$ along the $x$ - and $z$-axes depending on the distance $(s)$ between the point source and the grating. The diffraction pattern is calculated for illumination with a spherical wave at paraxial approximation [29] for $\lambda=$ $790 \mathrm{~nm}$. As can be seen, at $s=7 \mathrm{~cm}$ there is no Talbot plane, at $s=12 \mathrm{~cm}$ the plane is only one, whereas at $s=30 \mathrm{~cm}$ the Talbot planes are three. Hence, by using a divergent beam it is possible to ensure an equal contrast of fringes within a large $3 \mathrm{D}$ volume.

3.2. Experiment. To find the frequency content of the pattern created by a sinusoidal phase grating, we performed test measurements at different wavelengths using the optical arrangement in Figure 5. The divergent light beam (expanded by the lens L1) from the diode laser DL illuminates the grating SDG. The distance between the light source and the grating remained fixed. We recorded the FPs projected onto the ground glass screen GGS at varying distance $z$ (as indicated in Figure 5) for three different positions of the projector lens L2 with respect to the grating. Recording the phase gratings on a high-resolution holographic plate HP-650, laboratory production of CLOSPI-BAS (Bulgaria) was accomplished by an interferometric optical arrangement with an $\mathrm{He}-\mathrm{Ne}$ laser $(\lambda=632.8 \mathrm{~nm}, 30 \mathrm{~mW})$. An interference pattern was generated using an adjustable Michelson interferometer which provided equidistant sinusoidal fringes, whose period could vary in broad limits. The chemical processing of the holographic plates [30] was realized with a fixing developer which ensured formation of colloidal silver grains, practically phase recording, low level of noise, and high diffraction efficiency up to $70 \%$ in NIR. The required modulation of the recorded interference patterns was achieved by proper selection of exposures to fall into the dynamic range $0.5-$ $1.5 \mathrm{~mJ} / \mathrm{cm}^{2}$.

Figures 6(a), 6(b), 6(c), and 6(d) show 1D Fourier spectra of the projected fringes at $z=70 \mathrm{~cm}$ depending on the distance $d$ (see Figure 5) and the wavelength. Each spectrum is averaged over the whole registered FP. As can be seen, the frequency content of the FPs at different wavelengths is rather close for a divergent beam illumination. The distance $d=10 \mathrm{~cm}$ in Figure 5 seems to be optimal for generation of sinusoidal fringes.

\section{Systematic Errors of Phase Retrieval}

The analysis in the previous section shows that the systematic errors in phase determination are caused not only by the higher frequency content in the projected FPs but also by the fact that the spectra of the fringes generated at the four wavelengths differ from each other. This difference results in different contrast of the projected FPs, thus creating an additional source of errors. To evaluate the systematic error of phase retrieval, we simulated reconstruction of a plane surface from $M=4$ FPs by a four-step phase-shifting algorithm. The patterns were generated using the expression for the complex amplitude (6) at a given value of $z$. Deviation from a pure sinusoidal profile leads to periodical elevations and depressions in the reconstructed plane surface. Hence, to characterize the systematic error, we took the maximum deviation of the retrieved phase from its real value in radians, and plotted it as a function of $z$. Figure 7(a) illustrates the case when the phase is retrieved from four patterns generated at $\lambda=790 \mathrm{~nm}$ with plane-wave illumination, that is, when the error is induced only by the higher frequency 


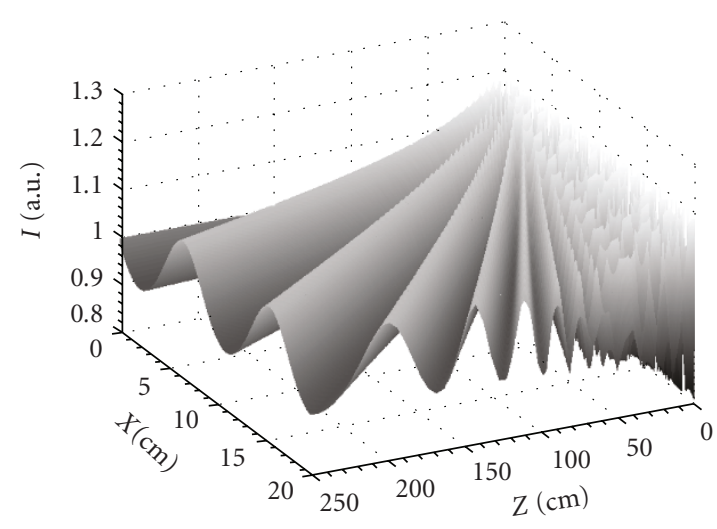

(a)

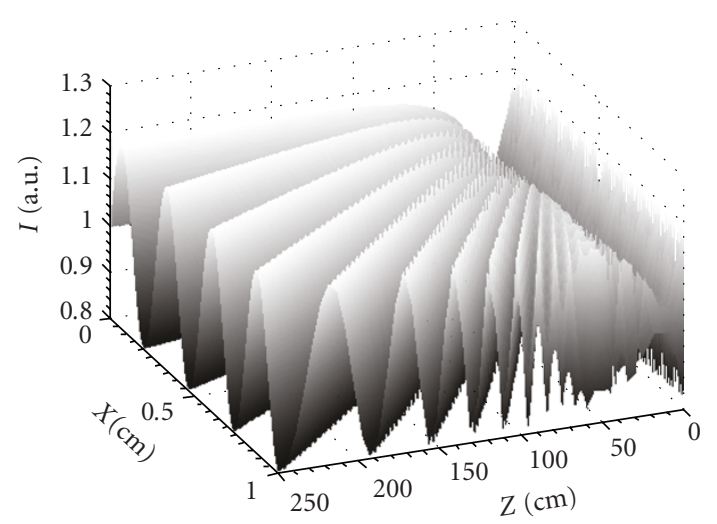

(c)

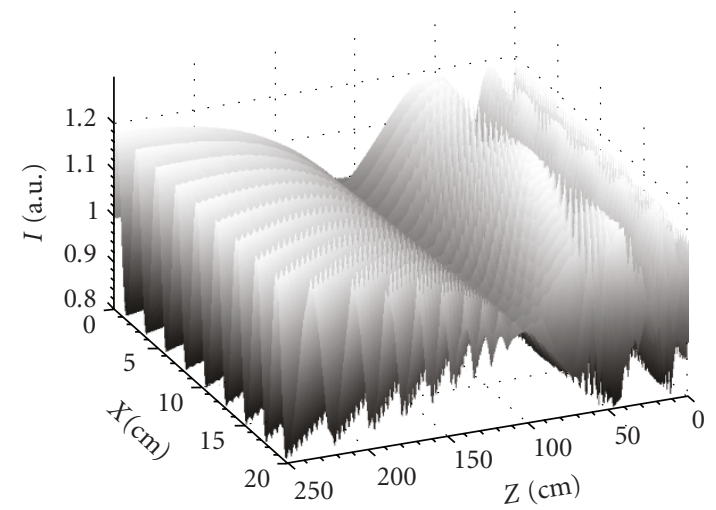

(b)

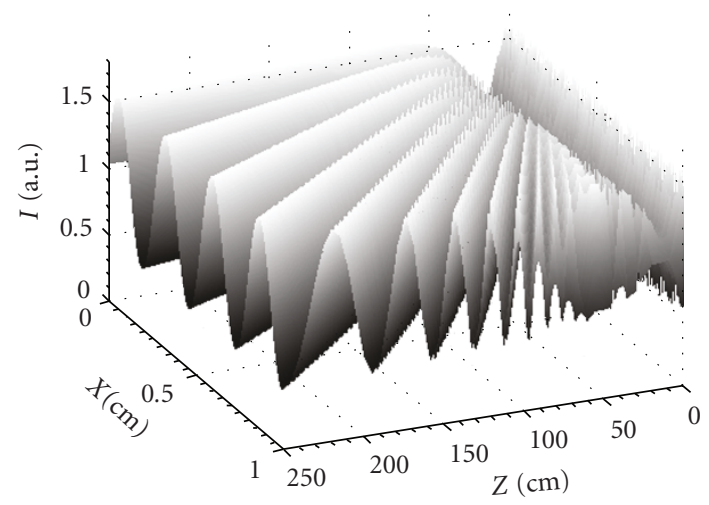

(d)

FIgURE 4: The intensity distribution of the light transmitted by a sinusoidal phase grating with $L=0.025 \mathrm{~cm}$ in the Fresnel zone for a spherical wave illumination: (a) $m=0.1, s=7 \mathrm{~cm}$; (b) $m=0.1, s=30 \mathrm{~cm}$; (c) $m=0.1, s=12 \mathrm{~cm}$; (d) $m=0.3, s=12 \mathrm{~cm}$.

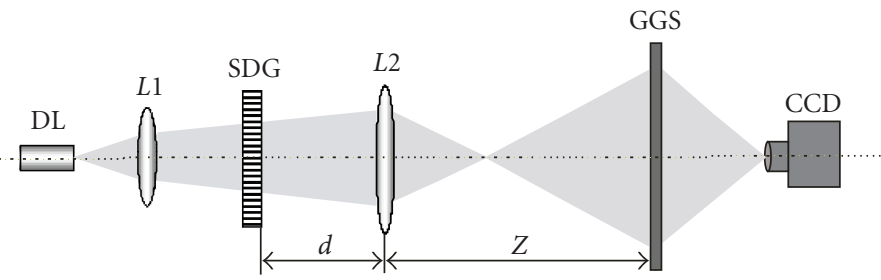

FIGURE 5: Optical arrangement for determination of frequency content of patterns projected by a sinusoidal phase grating —DL: diode laser, L1, L2: lenses, SDG: sinusoidal phase grating, GGS: ground glass screen.

content in the fringes. As can be seen, the error has a periodical distribution along the $z$-axis and is quite small for a modulation $m \leq 0.3$. Figure 7 (b) gives the systematic error in the case when the four FPs are generated at 790, 810, 850, and $910 \mathrm{~nm}$ for a plane-wave illumination. Comparison with the previous figure (Figure $7(\mathrm{a})$ ) clearly shows the substantial influence of the difference in the wavelengths on the accuracy of the phase retrieval; the error is acceptable only in a small area which includes the values of $z^{\prime \prime}$ for the considered wavelengths.
Fortunately, the result obtained with four-wavelength illumination with a spherical wave in paraxial approximation is more encouraging. The maximum deviation between the phase estimate and the real phase is depicted in Figure 8. As can be seen, the error does not exceed $0.06 \mathrm{rad}$ for $m=$ 0.3 within a very large range of distances from the grating. To explain why its value is so small, we include in the figure (Figure 8 ) the profiles of the four fringes projected at $790,810,850$, and $910 \mathrm{~nm}$ for $z=140 \mathrm{~cm}$ and $z=$ $230 \mathrm{~cm}$. The projected intensity distributions are very close, 

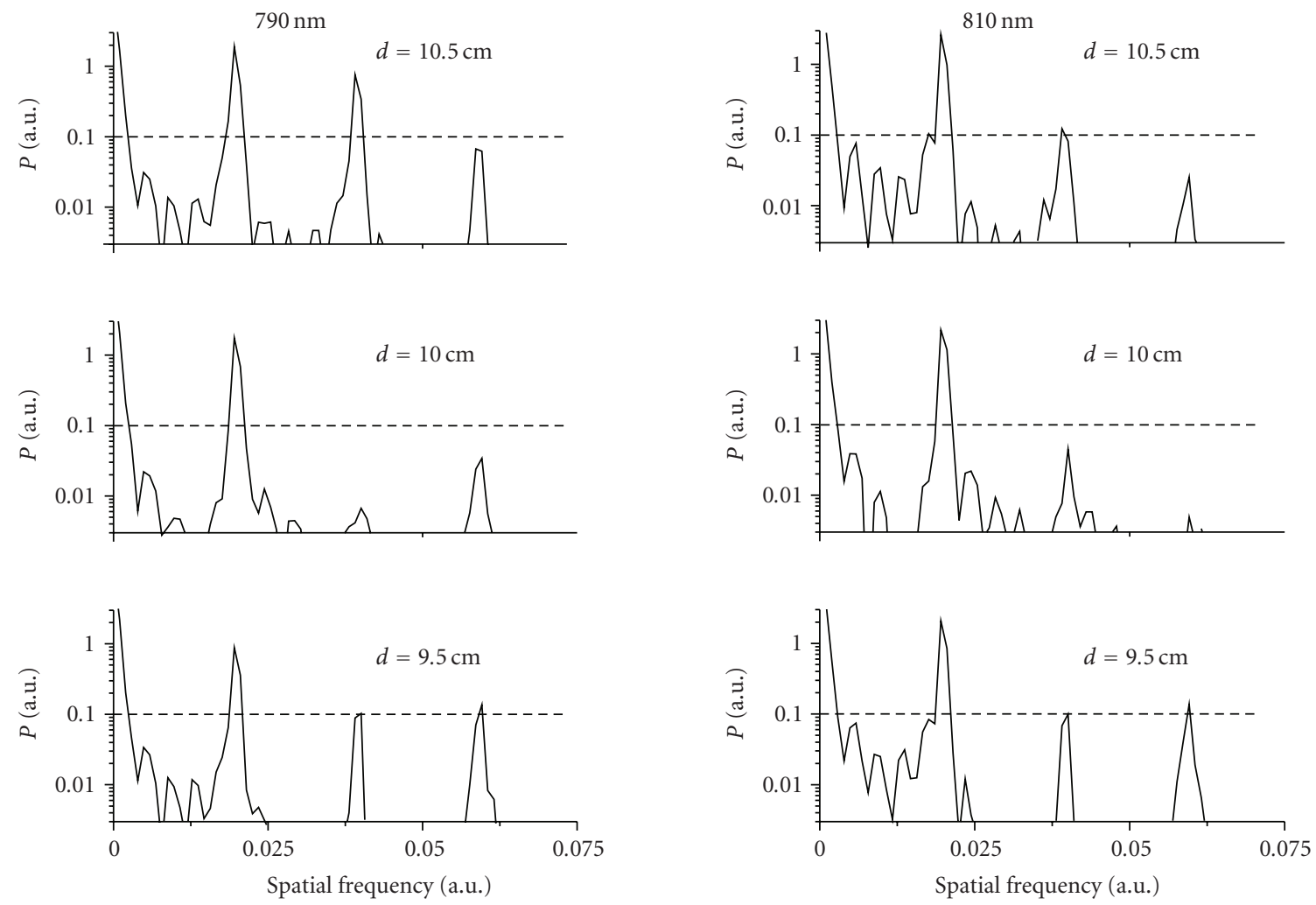

(a)

(b)
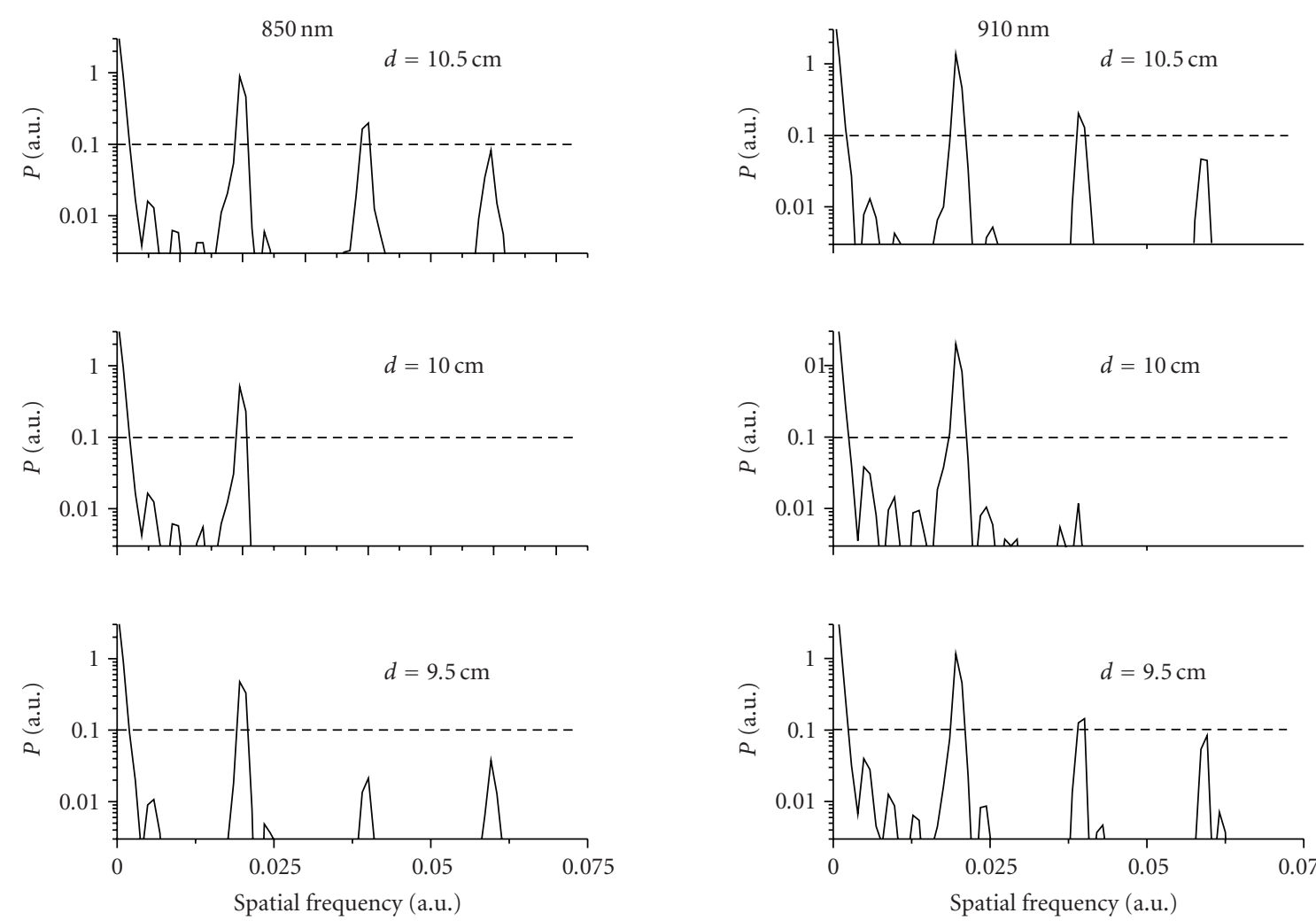

(c)

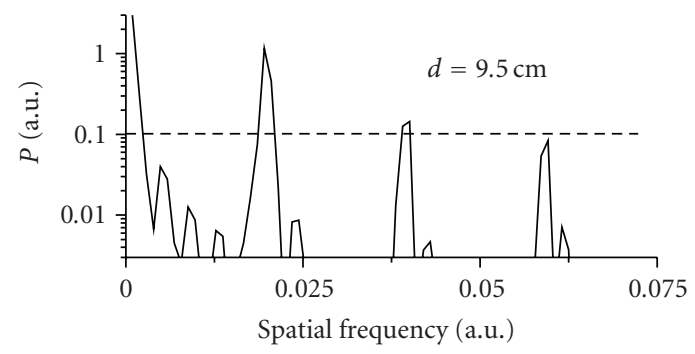

(d)

FIGURE 6: Frequency content of patterns created by a sinusoidal phase grating using an optical arrangement in Figure 5. 


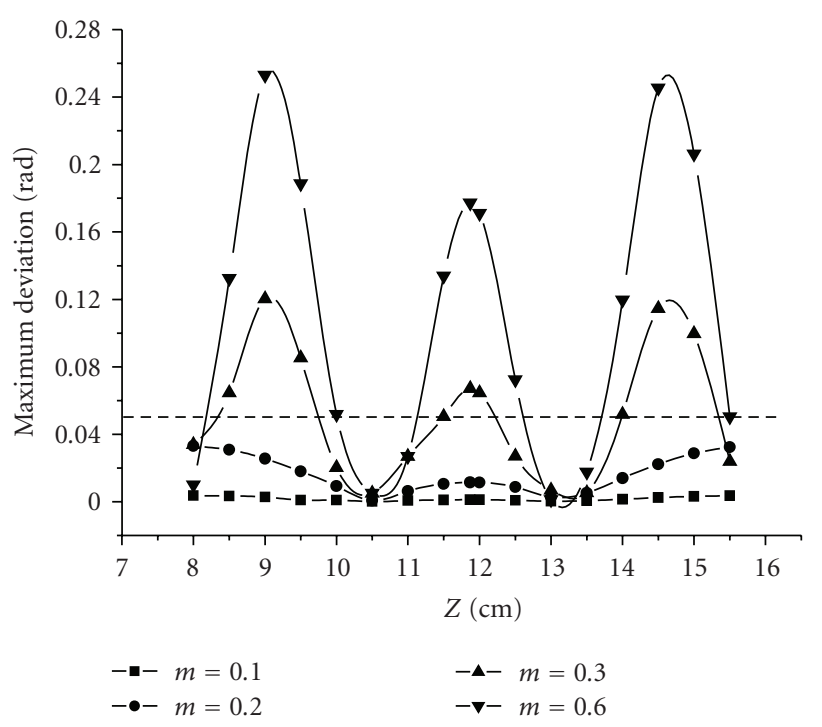

(a)

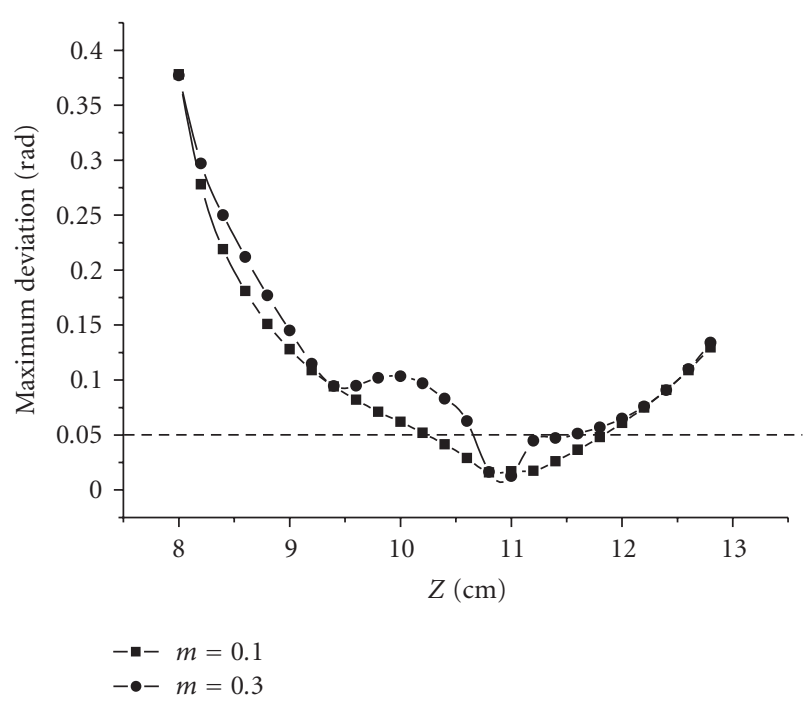

(b)

Figure 7: Maximum deviation of the difference between the phase estimate and the real-phase value for reconstruction of a plane surface at plane-wave illumination: (a) one-wavelength projection; (b) four-wavelength projection. Here, $z$ is the distance between the grating and the plane.

even at $m=0.3$. This result is confirmed by good quality of reconstruction of a plane from experimentally recorded patterns, as can be seen from the unwrapped phase map obtained for a GGS in Figure 5 at illumination with 790, 810, 850, and $910 \mathrm{~nm}$ (Figure 9). The FPs are recorded using only one CCD camera.

To prove the applicability of the multiwavelength approach with a sinusoidal phase grating as a projection element, we simulated reconstruction of a 3D surface of a dome in the case of the profilometric system in Figure 1 that represents a conventional cross-axes optical arrangement. The simulation includes registration of four FPs which are deformed by the object and four FPs of the reference plane

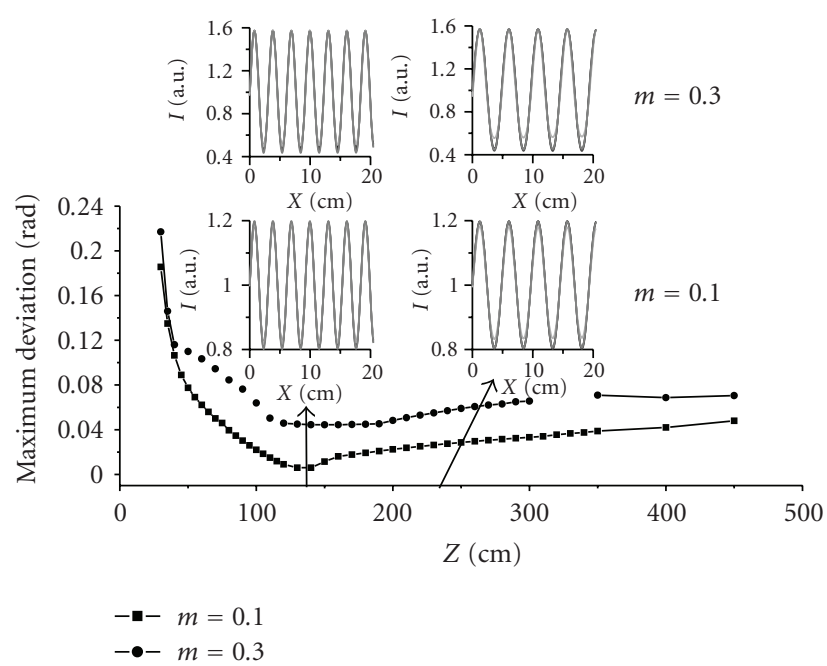

Figure 8: Maximum deviation of the difference between the phase estimate and the real-phase value for reconstruction of the plane surface from four fringe patterns recorded at four wavelengths with a spherical wave illumination (paraxial approximation); the plots in the inset give the lateral profile of the fringes generated at $\lambda=790$, 810,850 , and $910 \mathrm{~nm}$. Here, $z$ is the distance between the grating and the plane.

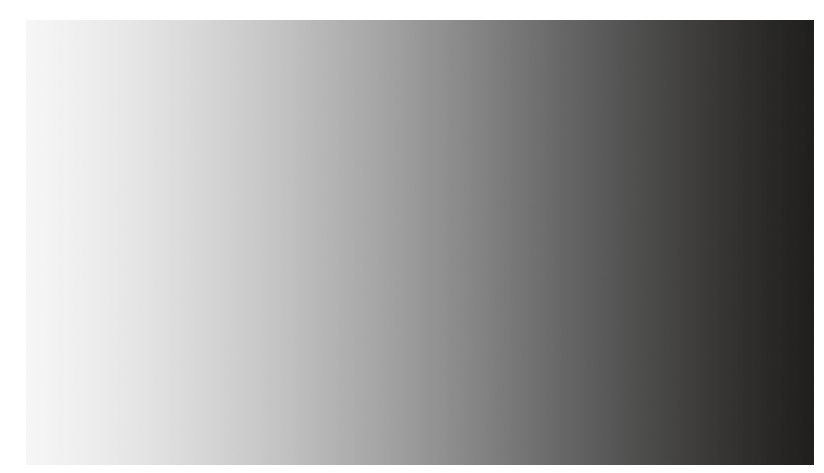

FIGURE 9: Unwrapped phase map of a plane that is retrieved from four fringe patterns projected by using four identical sinusoidal gratings and four diode lasers emitting at 790, 810, 850, and $910 \mathrm{~nm}$.

without the object at the following values of the system parameters: $L=0.025 \mathrm{~cm}, \lambda_{1}=790 \mathrm{~nm}, \lambda_{2}=810 \mathrm{~nm}, \lambda_{3}$ $=850 \mathrm{~nm}, \lambda_{4}=910 \mathrm{~nm}$, in addition to 512 pixels along the $x$ - and $y$-axes and normal viewing directions with respect to the reference plane of all four CCD cameras which capture the same part of the object. The angle between the axes of the projection and recording optical systems is 30 . The dome parameters are as follows: the radius on the reference plane is 254 pixels and the maximum dome height at the center is 90 pixels. The $3 \mathrm{D}$ surface reconstruction included the following steps: (i) calculation of the wrapped phase maps by using formula (2) from the FPs recorded with the object and the reference plane alone, respectively; (ii) unwrapping 
TABLe 1: Maximum negative and positive deviations in \% from the dome height.

\begin{tabular}{lrrrr}
\hline Distance $z(\mathrm{~m})$ & \multicolumn{3}{c}{ Modulation parameter $m$} & 0.3 \\
\hline \multirow{2}{*}{1.3} & 0.1 & 0.2 & -0.75 & -1.19 \\
& -0.18 & -0.55 & 0.81 & 0.96 \\
1.5 & 0.42 & 0.54 & -0.79 & -1.27 \\
& -0.26 & -0.6 & 0.82 & 1.07 \\
1.8 & 0.41 & 0.46 & -1.14 & -1.41 \\
& -0.49 & -0.77 & 0.96 & 1.52 \\
2.4 & 0.57 & 0.72 & -1.53 & -1.98 \\
& -0.81 & -1.11 & 1.54 & 1.96 \\
\hline
\end{tabular}

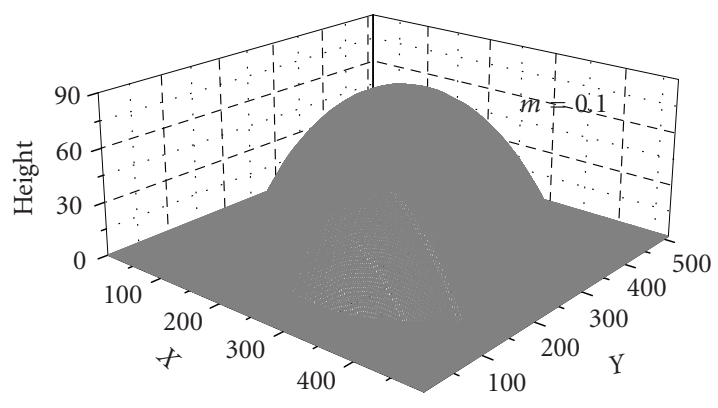

(a)

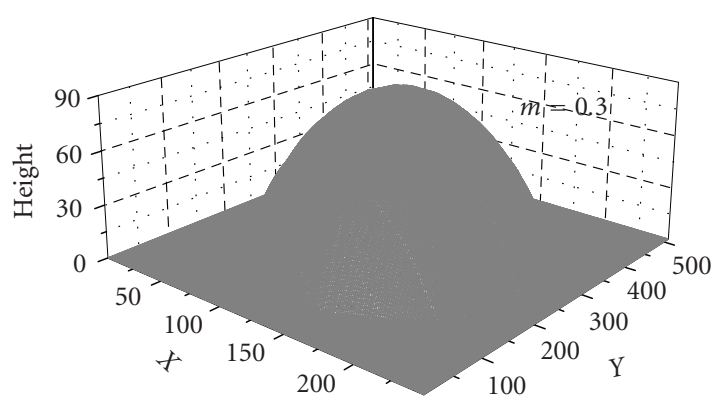

(b)

FIGURE 10: Reconstruction of a 3D surface by a phase-shifting technique from four fringe patterns generated with four-wavelength divergent illumination. The projection distance is $z=1.3 \mathrm{~m}$; the grating spacing is $L=0.025 \mathrm{~cm}$.

by a quality-guided algorithm [30] of both phase maps; (iii) subtraction of the unwrapped phase map corresponding to the reference plane from the unwrapped phase map corresponding to the object, and, finally, (iv) calculation of the $3 \mathrm{D}$ coordinates. The projected FPs are simulated for a spherical wave illumination in paraxial approximation. Simulation was made without any noise, and so no filtration is applied to the images. Figure 9 depicts two reconstructions of the dome for $m=0.1$ and $m=0.3$. Small ripples can be noticed on the dome surface close to its apex for the latter case. We evaluated the accuracy of reconstruction for the distances within the interval from $1.3 \mathrm{~m}$ up to $2.4 \mathrm{~m}$ which corresponds to the smallest systematic errors in
Figure 8 . Table 1 gives the maximum values of the positive and negative deviations of the reconstructed surface from the real dome; the deviations are normalized to the dome height and multiplied by $100 \%$. The obtained values of maximum deviations on the order of $0.5-2 \%$ prove that usage of a sinusoidal grating ensures acceptable accuracy of the profilometric measurement.

\section{Conclusion}

Fringe projection profilometric system which combines single-shot acquisition with phase-shifting processing algorithm for "real-time" surface measurement is presented. Sinusoidal phase gratings and single-mode diode lasers emitting at four different wavelengths of $\lambda_{1}=790 \mathrm{~nm}, \lambda_{2}=$ $810 \mathrm{~nm}, \lambda_{3}=850 \mathrm{~nm}, \lambda_{4}=910 \mathrm{~nm}$ are used. The main advantages of coherent illumination by NIR diode lasers are higher efficiency, larger focal depth, and fringes contrast, which lead to higher sensitivity and accuracy of measurement. Careful maintaining of grating modulation could ensure vanishing contribution of higher diffraction orders. The performance of the proposed four-wavelength system was checked by simulation of reconstruction of a plane and a 3D object (dome) for four identical gratings with $L=0.025 \mathrm{~cm}$ at different values of the grating modulation parameter. The problem of higher harmonics influence has been thoroughly analyzed. Both cases of collimated and divergent beam illumination have been studied. The results were experimentally verified. The analysis made confirms that the sinusoidal phase grating can serve as a projection element in a profilometric system as well as being used at multiwavelength illumination.

\section{Acknowledgment}

This work is supported by EC within FP6 under Grant no. 511568 .

\section{References}

[1] F. Chen, G. M. Brown, and M. Song, "Overview of threedimensional shape measurement using optical methods," Optical Engineering, vol. 39, no. 1, pp. 10-22, 2000. 
[2] T. Kreis, "Computer-aided evaluation of holographic interferograms," in Holographic Interferometry: Principles and Methods, P. K. Rastogi, Ed., , pp. 151-212, Springer, Heidelberg, Germany, 1994.

[3] B. V. Dorrío and J. L. Fernández, "Phase-evaluation methods in whole-field optical measurement techniques," Measurement Science and Technology, vol. 10, no. 3, pp. R33-R55, 1999.

[4] S. Zhang and S.-T. Yau, "High-resolution, real-time 3D absolute coordinate measurement based on a phase-stepping method," Optics Express, vol. 14, no. 7, pp. 2644-2654, 2006.

[5] M. Takeda, H. Ina, and S. Kobayashi, "Fourier-transform method of fringe-pattern analysis for computer-based tomography and interferometry," Journal of the Optical Society of America, vol. 72, pp. 156-160, 1982.

[6] M. Takeda and K. Mutoh, "Fourier transform profilometry for the automatic measurement of 3-D object shapes," Applied Optics, vol. 22, no. 24, pp. 3977-3982, 1983.

[7] T. Kreis, "Digital holographic interference-phase measurement using the Fourier-transform method," Journal of the Optical Society of America A, vol. 3, no. 6, pp. 847-855, 1986.

[8] J. Zhong and J. Weng, "Spatial carrier-fringe pattern analysis by means of wavelet transform: wavelet transform profilometry," Applied Optics, vol. 43, no. 26, pp. 4993-4998, 2004.

[9] L. R. Watkins, "Phase recovery from fringe patterns using the continuous wavelet transform," Optics and Lasers in Engineering, vol. 45, no. 2, pp. 298-303, 2007.

[10] M. Servin and F. J. Cuevas, "A novel technique for spatial phase-shifting interferometry," Journal of Modern Optics, vol. 42, no. 9, pp. 1853-1862, 1995.

[11] M. Servin, J. L. Marroquin, and F. J. Cuevas, "Demodulation of a single interferogram by use of a two-dimensional regularized phase-tracking technique," Applied Optics, vol. 36, no. 19, pp. 4540-4548, 1997.

[12] P. H. Chan, P. J. Bryanston-Cross, and S. C. Parker, "Fringepattern analysis using a spatial phase-stepping method with automatic phase unwrapping," Measurement Science and Technology, vol. 6, no. 9, pp. 1250-1259, 1995.

[13] C. Quan, C. J. Tay, L. Chen, and Y. Fu, "Spatial-fringemodulation-based quality map for phase unwrapping," Applied Optics, vol. 42, no. 35, pp. 7060-7065, 2003.

[14] C. J. Tay, C. Quan, F. J. Yang, and X. Y. He, "A new method for phase extraction from a single fringe pattern," Optics Communications, vol. 239, no. 4-6, pp. 251-258, 2004.

[15] C. Quan, C. J. Tay, F. Yang, and X. He, "Phase extraction from a single fringe pattern based on guidance of an extreme map," Applied Optics, vol. 44, no. 23, pp. 4814-4821, 2005.

[16] M. de Angelis, S. De Nicola, P. Ferraro, A. Finizio, S. Grilli, and G. Pierattini, "Profile measurement of a onedimensional phase boundary sample using a single shot phasestep method," Optics and Lasers in Engineering, vol. 43, no. 12, pp. 1305-1314, 2005.

[17] K. Creath, "Phase-shifting holographic interferometry," in Holographic Interferometry: Principles and Methods, P. K. Rastogi, Ed., pp. 109-150, Springer, Heidelberg, Germany, 1994.

[18] O. A. Skydan, M. J. Lalor, and D. R. Burton, "Technique for phase measurement and surface reconstruction by use of colored structured light," Applied Optics, vol. 41, no. 29, pp. 6104-6117, 2002.

[19] O. A. Skydan, M. J. Lalor, and D. R. Burton, "Using coloured structured light in 3-D surface measurement," Optics and Lasers in Engineering, vol. 43, no. 7, pp. 801-814, 2005.

[20] S. Yoneyama, Y. Morimoto, M. Fujigaki, and Y. Ikeda, "Three-dimensional surface profile measurement of a moving object by a spatial-offset phase stepping method," Optical Engineering, vol. 42, no. 1, pp. 137-142, 2003.

[21] V. Sainov, E. Stoykova, and J. Harizanova, "Real time phase stepping pattern projection profilometry," in Speckles, From Grains to Flowers, vol. 6341 of Proceedings of SPIE, pp. 1-6, Nimes, France, September 2006.

[22] Y. Bitou, "Digital phase-shifting interferometer with an electrically addressed liquid-crystal spatial light modulator," Optics Letters, vol. 28, no. 17, pp. 1576-1578, 2003.

[23] K. Hibino, B. F. Oreb, D. I. Farrant, and K. G. Larkin, "Phase shifting for nonsinusoidal waveforms with phase-shift errors," Journal of the Optical Society of America A, vol. 12, no. 4, pp. 761-768, 1995.

[24] H. Liu, G. Lu, S. Wu, S. Yin, and F. T. S. Yu, "Speckle-induced phase error in laser-based phase-shifting projected fringe profilometry," Journal of the Optical Society of America A, vol. 16, no. 6, pp. 1484-1495, 1999.

[25] A. Styk and K. Patorski, "Identification of nonlinear recording error in phase shifting interferometry," Optics and Lasers in Engineering, vol. 45, no. 2, pp. 265-273, 2007.

[26] G. Schirripa Spagnolo, D. Ambrosini, and D. Paoletti, "Displacement measurement using the Talbot effect with a Ronchi grating," Journal of Optics A, vol. 4, no. 6, pp. S376-S380, 2002.

[27] J. Goodman, Introduction to Fourier Optics, McGraw-Hill, Boston, Mass, USA, 1996.

[28] B. F. Oreb and R. G. Dorsch, "Profilometry by phase-shifted Talbot images," Applied Optics, vol. 33, no. 34, pp. 7955-7962, 1994.

[29] K. Patorski and S. Kozak, "Self-imaging with nonparabolic approximation of spherical wave fronts," Journal of the Optical Society of America A, vol. 5, pp. 1322-1327, 1988.

[30] D. Ghiglia and M. Pritt, Two-Dimensional Phase Unwrapping: Theory, Algorithms, and Software, John Wiley \& Sons, New York, NY, USA, 1998. 